\title{
Trauma Tapping Technique: Practical First Aid for Stress and Trauma
}

\author{
Marie Claire Gasanganwa ${ }^{1}$, Rebecca L White ${ }^{1}$ \\ ${ }^{1}$ University of Rwanda, College of Medicine and Health Sciences, Rwanda
}

\section{Background}

Epidemiological studies on posttraumatic stress disorder (PTSD) show a lifetime prevalence rate of up to $6.8 \%$. PTSD symptoms include re-experiencing the traumatic event, avoidant behavior for the memories of the event, and hyperarousal. By 2020, psychological trauma will be among the leading causes of global disability, alongside depression and heart disease. A post-genocide study conducted in 2009 by the Ministry of Health $(\mathrm{MoH})$ in Rwanda showed that PTSD prevalence was $28.5 \%$ in the adult population, and $53.9 \%$ of these cases had a comorbid depression. Providing community outreach programs at a primary care level is one of the major goals of the University of Rwanda. The Ministry of Health emphasizes the integration of mental health services into general medical care at a grass roots level. Recognizing the role of psychotrauma on physical health, the Ministry wants to implement simple but effective community mental health treatments.

\section{Methods}

Trauma tapping technique (TT'T) is a procedure that uses touch through tapping to relieve anxiety symptoms. T'T'T is a nonverbal process that can be delivered at minimal cost with the potential for easy dissemination. This technique can be applied in urban or rural communities and in medical or mental health environments.

\section{Results}

Psychotrauma has resulted in a host of mental health issues such as PTSD, anxiety and depression. Many Rwandan families experience these conditions. TTT has been shown to be useful in anxiety, PTSD and pain control arenas.

\section{Conclusion}

Trauma Tapping Technique is an emerging psychological intervention that has been used to treat a variety of conditions, including exam stress and public speaking anxiety, as well as PTSD. TT'T may be a feasible initial community based treatment for psychotrauma, PTSD and anxiety disorders in Rwanda. This nonverbal intervention has the potential to augment mental health care.

Key words: posttraumatic stress disorder, trauma tapping therapy, nonverbal communication, genocide, mental health Rwanda 